\title{
THE FEATURES OF THE MORPHOLOGY CRYSTALLINE INCLUSIONS AND THE REAL STRUCTURE OF DIAMOND
}

\author{
Y.P. Barashkov, S.B. Talnikova
}

Yakut. Institute of Geosciences, 39, Lenin Pr., Yakutsk, 677891, Republic Sakha, Russia

The morphologic varieties of crystal inclusions in diamonds have been studied quite sufficiently and the results of such studies are comprehensively covered in various publications. The external form of syngenetic inclusions in diamonds is a classic example of "supressed" growth forms. It has been determined that in the majority of cases the morphology of inclusions is entirely induced by the diamond itself and that it does not depend on the mineral type and the syngony of the enclosed mineral (Sobolev et all, 1972, Bartoshinsky et all, 1980). Depending on the degree of form distortion, the following three types of crystal inclusions in diamonds are distinguished: pseudoprismatic, pseudo-tabular and pseudo-octahedral.

As shown by goniometric measuring and studies of internal structure of natural diamonds, the face of an octahedron $\{111\}$ is the only active form of crystal growth, which has its own pyramid of growth. All other flat surfaces are not faces from the point of view of crystallography. Such simple figures as a cube $\{100\}$, a rhombic dodecahedron $\{110\}$, a trigonomic trioctahedron $\{113\}$ and others are in factin hibitory planes because they do not have their own pyramids of growth and are formed when octahedron faces check out. According to the fundamentals of crystallography, the growth of a crystal is registered only if the incorporation of a particle (an atom or a molecule) does not affect the energy of the surface. According to the Hartman-Purdock theory ofperiodic correlation chains, octahedral faces in a diamond's crystal lattice in the presence of enighboring minerals are defined as "atom smooth" F-faces, while S-(110) and K-(100) faces are defined as "atom grainy" faces. New particles join "atom smooth" surfaces at fissures which are clustered at levels, which are generated by various sources. As a result of this the level is tangentially moving along the plane surface. In this situation the growth of a crystal takes place layer by layer, and, accordingly, the smooth faces grow respectively (the so-called layered or tangential growth mechanism).

The macro-morphology of a crystal, the relief of faces give us reason to define the atom mechanism, the cynetics and the processes of layered or normal growth of a crystal. The consecutive reduction of face surfaces of a natural diamond octahedron, formed by layers of various relieves (from finest to the grainiest), allows us to define the layered mechanism of crystal growth. The layers are generated in the central part of the face and grew from the center to the periphery. In this case the speed of the crystal growth is defined by the cynetics of processes on the surface of face and does not depend on the speed of atom diffusion. It follows that octahedral diamonds grew in the cynetic mode of crystallization.

The study of crystal inclusions in flat-parallel planes, cut out of diamond octahedrons (110), allows us to reveal the topography of the enclosed mineral in correlation to certain diamond growth sectors and zones. We have established a correlation between the morphology of mineral inclusions and the real structure of diamonds which contain them. The above three main types of crystal inclusions are orderly oriented in accordance with a diamond symmetry elements and are closely correlated to its internal structure.

Pseudo-prismatic inclusions are always oriented longitudinally along one of the diamond's octahedron edges. The pseudo-faces of inclusions, the parallel faces $\{111\}$ of a diamond are well developed and flat. The induced hatching on such faces is not observed, as a rule. This is, evidently, 
explained by the fact that the deviation of induced faces from average plane of a pseudo-face $\{111\}$ is minor and that the subsequent consolidation of the induced surface took place. The consolidation of induced faces, which form the curvilinear surface at the spot of pseudo-edges, most adequately explains the observed curve of the tops and edges of such inclusions and their flowed surface.

Pseudo-tabular inclusions have very well developed opposite faces, which lie in the octahedron plane (111). The flattening of such inclusions is perpendicular to one of the triplex axes of a diamond crystal. Other pseudo-faces are less well developed and often have induced faces. Considerably flattened tabular inclusions can be also referred to this morphological variety. The most well developed pseudo-faces do not always refer to the same crystallographic zone. An olivine inclusion of a tabular shape shows that the induced faces belong to at least two crystallographic zones. In such cases the neighboring pseudo-faces are projected to the same plane.

Inclusions of the pseudo-octahedral type are shaped as inverted diamond crystals (see Fig.). The pseudo-faces and pseudo-edges of the enclosed mineral are regularly oriented relatively the respective faces and edges of the host diamond. Some pseudo-faces of inclusions are covered by fine induced hatching. But in most cases octahedral pseudo-faces of inclusions have a smooth and plane surface. In this case the deviation of induced faces from the average surface of inclusion's octahedral pseudo-face was insignificant and was followed by the consequent leveling of its relief. The appearance of roundish shape of inclusions is adequately explained by the fusion of induction faces, which form the curvilinear surface at the octahedron's pseudo-edges.
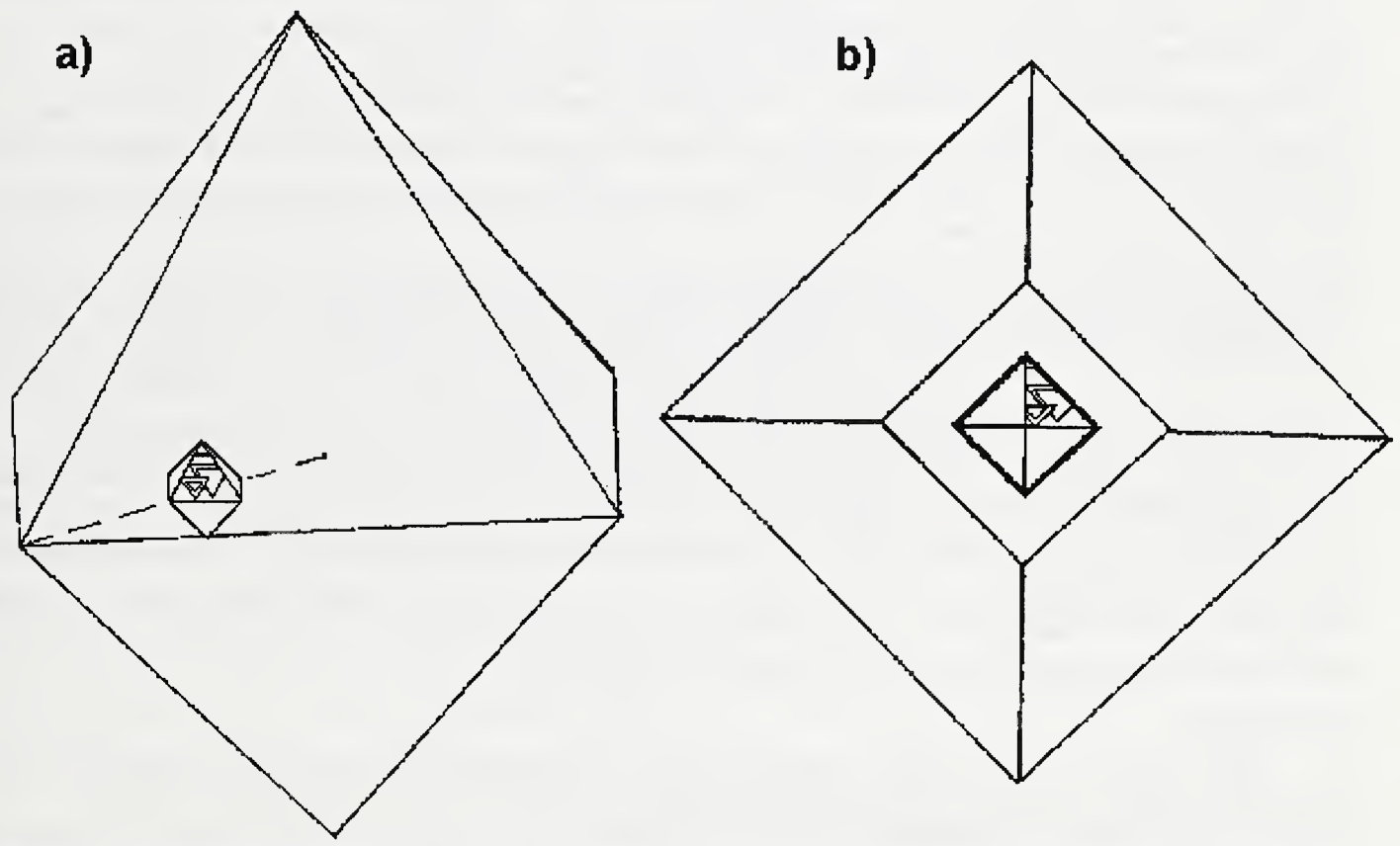

Figure: Schematic drawing clinopyroxene Inclusion of octahedral shape in a diamond. a) projection to a plane (111). The dotted line shows the diamond crystal summit movement line. b) projection of a plane (100). The top of the crustal which "captured" the inclusions is ground along (100).

Among inclusions of pseudo-octahedral type we can find hemimorphous crystals which have half of the most well developed octahedron's faces, as a result of which they look like pyramids. Inclusions 
of cubic octahedral type with polycentric formation of faces represent a variety of inclusions of pseudo-octahedral type (Sobolev et all, 1983).

For the first time we carried out a study of inclusions in such morphological varieties of diamonds as dodecahedrons and cubes. The visually observed rough zonal coloring in cubic diamonds of the 2-nd and 3-rd mineral varieties is caused by micro-inclusions, which concentrate in the zones and sectors [100], giving them darker color. These micro-inclusions are located in three interperpendicular planes (100), forming a sagenite-like lattice.

Attention should be paid to clusters of two and even three mineral inclusions which have the shape of a monolithic crystal. We observed inclusions of pseudo-octahedral shape which consisted of garnets and pyroxenes, sulfides and silicates, chromite and pyroxenes. The accretion surface of these minerals is always smooth, without any traces of concurrent growth, while the accretion surface of such clustered inclusions with the diamond is, undoubtedly, in induced. The issue of formation of such inclusions and the prevalence of inverted diamond prints in them is still open to discussion.

Thus, the analysis of the morphology of syngenetic inclusions in diamonds shows the predominant development of octahedral faces. Let us discuss the possible mechanism of formation of such inclusions. As we know, the growth elements of real crystals are pyramids of face growth, the planes of edge movement and the summit movement lines. Accordingly, we can identify respective forms of crystals which can be positive and inverted. In the process of growth the diamond crystal "ousts" the neighboring growing mineral. The surface of contact between the mineral and the diamond is characterized by slower growth, this is why the diamond grows in this zone slower than in other zones, forming an "indent" - a negative growth element. Depending on which part of the diamond's surface contacted with the growing mineral, the diamond can register negative values of face $\{111\}$, edge $<110>$ or summit [100] growth. These negative growth elements "capture" the repelled syngenetic minerals.

The above gives us reason to conclude that there is a connection between the external morphology of syngenetic inclusions and the elements of a diamond's real structure. "Captured" minerals of pseudo-tabular type are correlated with pyramids of face growth $\{111\}$, minerals of pseudoprismatic type - with the planes of edge movement (110) and inclusions of pseudo-octahedral type with summit movement lines [100]. The fact that the inverted shape of syngenetic inclusions in diamonds is caused by growth processes is partially confirmed by the results of experiments on recrystallization of diamonds. These experiments yielded diamonds which contain inclusions of alloys of $\mathrm{Ni}$ and $\mathrm{Mn}$ and a mixture of $\mathrm{CO}_{2}, \mathrm{H}_{2}, \mathrm{~N}_{2}$ and others, which have a shape of inverted diamond crystals (Bakumenko et all, 1984). The inclusions components register the composition of the environment in which the experiments were carried out.

\section{References}

Z.V. Bartoshinsky et all, 1980. Crystallographic morphology of garnet inclusions in natural diamonds, geology and geophysics, No. 3, pp. 12-22

N.V. Sobolev et all, 1972, Crystal inclusions of octahedral shape in diamonds, Reports of USSR Academy of Sciences, Vol. 204, No. 1, pp. 192-195

N.V. Sobolev et all, 1983, Eclogitic paragenesis of diamonds from "Mir" kimberlite pipe": Mantle xenolites and the problem of ultrabasic magmas", Novosibirsk, Nauka, pp. 44-16.

I.T. Bakumenko et all, 1984, Faceted inclusions in diamond crystals, Reports of USSR Academy of Sciences, Vol. 278, No. 6, pp. 1461-1465 